\title{
The Definition of Persistent Pulmonary Hypertension of the Newborn (PPHN) in randomised controlled trials: a systematic literature review

\section{Background}

Persistent pulmonary hypertension of the newborn (PPHN) is a severe cardiorespiratory disorder in term and late preterm infants affecting around 2 per 1000 live births. It is frequently associated with hypoxaemic respiratory failure complicating disorders such as surfactant-deficient lung disease, meconium aspiration syndrome, congenital diaphragmatic hernia and sepsis.

The effectiveness of inhaled nitric oxide (iNO) in improving oxygenation in neonates with hypoxaemic respiratory failure is well established. iNO is licensed in newborn infants $\geq 34$ weeks' gestation with hypoxic respiratory failure associated with clinical or echocardiographic of pulmonary hypertension in order to improve oxygenation and to reduce the need for ECMO. However, its use in preterm infants $<34$ weeks gestation is 'off-label'. There is emerging evidence that PPHN physiology confirmed echocardiographically is a key determinant of the response to iNO and short- and long-term outcome.

In 1969, Gersony and colleagues published case reports of two neonates with pulmonary hypertension and described this condition as 'persistent fetal circulation'. However, in contrast to adults and older children, there is no accepted international definition of pulmonary hypertension or PPHN in neonates.

\section{Aim}

The aim of this study was to systematically review the clinical and echocardiographic parameters used to define persistent pulmonary hypertension (PPHN) in published randomised controlled trials of iNO in neonates.

\section{Methods}

We searched PubMed, the Cochrane Library and clinical trials registries to identify all randomised controlled trials evaluating the use of iNO in the newborn infants. A pre-defined search strategy was used to identify potentially-eligible trials. Only full, peer-reviewed English language publications were considered. Two reviewers (SS and $\mathrm{EH}$ ) independently assessed each publication to determine eligibility. A third reviewer (NS) verified that the entry criteria had been met and resolved any disagreements between the two original reviewers. Any duplicate or linked publications were excluded.

Each published trial was examined in detail to determine whether pulmonary hypertension was included as a criterion for eligibility for trial entry and, if so, what clinical and/or echocardiographic definitions were used.

\section{Results}

A total of 1021 trials of iNO therapy in neonates were identified from PubMed (590 hits), the Cochrane Library (361 hits) and ClinicalTrials.gov (70 hits). After exclusion of duplicate/linked publications, 998 trials were considered for further review.

Of these, 33 were eligible for inclusion within this systematic review; 24 were trials of iNO versus placebo/no treatment and 9 were comparative trials of iNO and another vasodilator.

Table 1: Criteria used to define PPHN

\section{Criteria}

Oxygenation Index (OI)

Number of studies $(n=33)$

Echocardiographic indices to diagnose PPHN

18

Chest X-ray findings

19

Respiratory support parameters

28

Combination of above criteria

\section{Results}

Table 2 -Echocardiographic criteria use to define pulmonary hypertension

Echocardiographic indices used to define pulmonary hypertension

Total number of studies $(n=19)$

Right-to-left/bi-directional flow at ductal

and/or atrial leve

14

Characteristics of interventricular septum

5

Absolute measurement of systolic PAP

Assessment of RV size/function

Presence of TR (but systolic PAP not

specified)

Echocardiographic indices not specified

11

12

3

Note: most trials used a combination of echocardiographic indices to define pulmonary hypertension but there was no uniformity in which indices were used.

\section{Key Findings}

The majority of trials used a combination of clinical and echocardiographic criteria.

- Various clinical and echocardiographic criteria were used to define pulmonary hypertension.

- There was no consistency in echocardiographic indices chosen to define pulmonary hypertension

Figure 1 -Common echocardiographic techniques to assess pulmonary hypertension - V max of tricuspid regurgitation and ductal flow characteristics
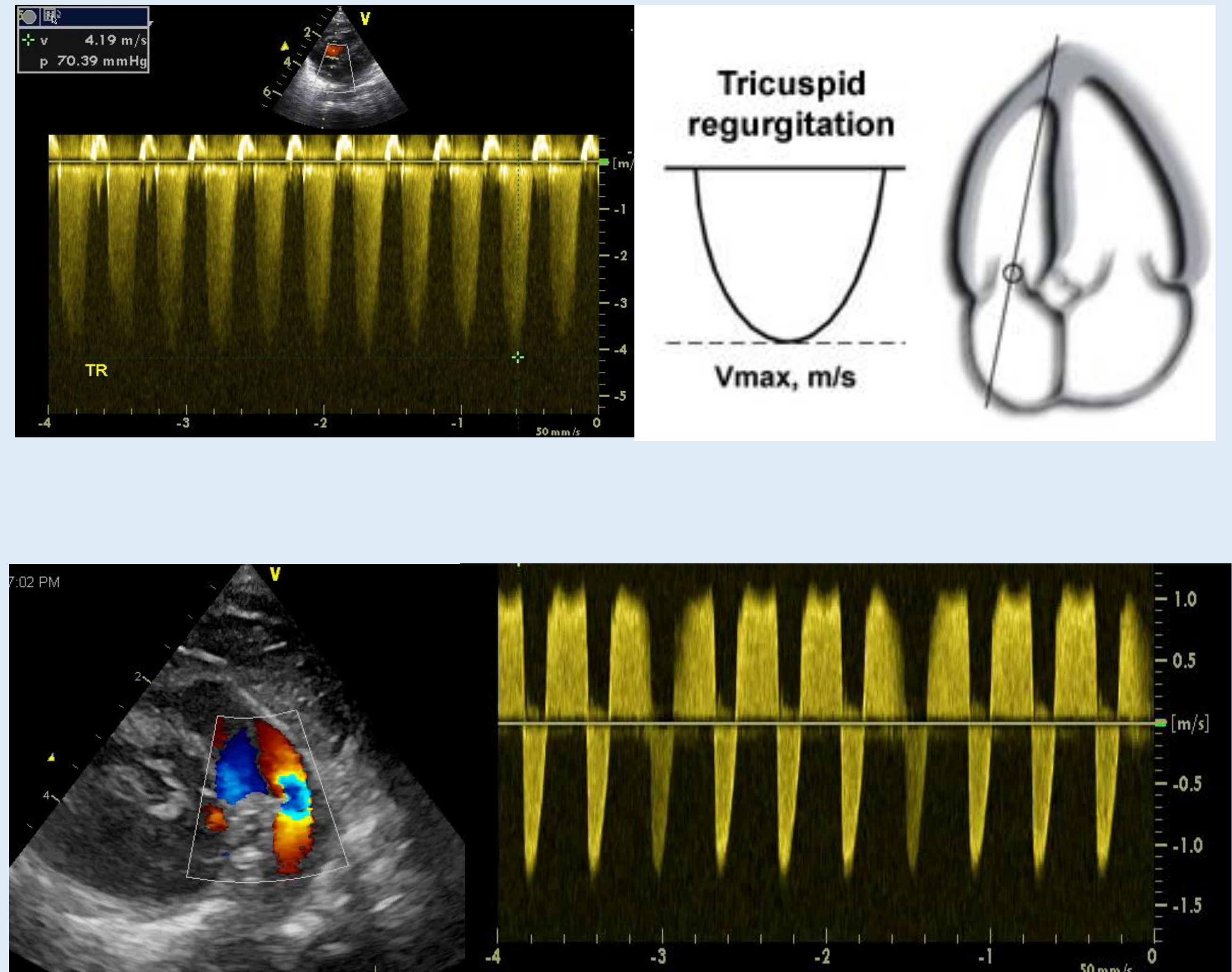

\section{Conclusions}

1. This study found wide variation in the clinical and echocardiographic criteria used to define pulmonary hypertension in newborn infants.

2. The lack of an accepted standardised definition of neonatal pulmonary hypertension makes comparison of studies difficult.

3. Establishing an international consensus definition of PPHN would help to design and interpret future clinical trials in this area.

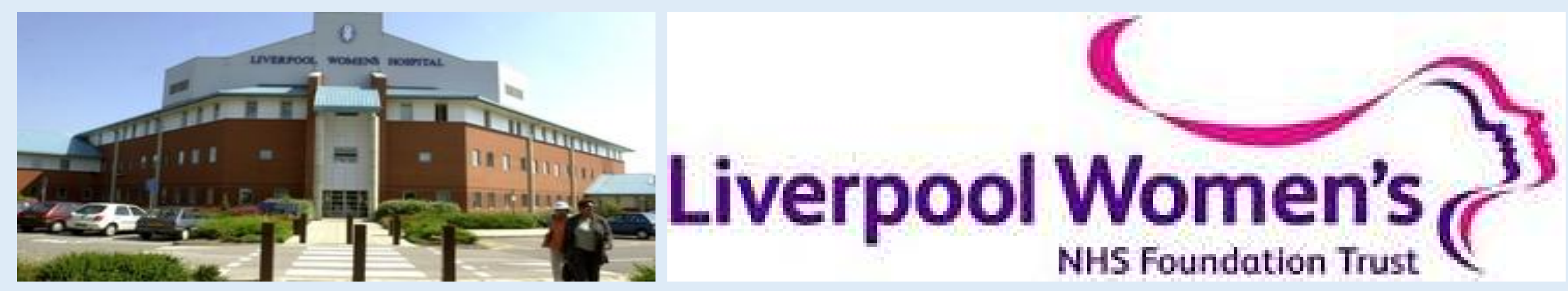

